FNG \& Demiroğlu Bilim Tıp Transplantasyon Dergisi 2018;3(1-2):42-46

doi: 10.5606/fng.transplantasyon.2018.010

\title{
Uterus nakli
}

\section{Uterus transplantation}

\author{
Mine Ün ${ }^{1}(\mathbb{D}$, Oytun Erbaş²i \\ ${ }^{1}$ İstanbul Aydın Üniversitesi Tip Fakültesi Öğrencisi, İstanbul, Türkiye \\ ${ }^{2}$ Demiroğlu Bilim Üniversitesi Tip Fakültesi Fizyoloji Anabilim Dal, İstanbul, Türkiye
}

\begin{abstract}
öz
Canlı donörlerden alıcılara yapılan uterus nakilleri sayesinde başarılı doğumlar gerçekleşmiștir. İşlevsiz bir uterus nedeniyle kısır olan kadınlar tedavi edilebilmektedir. Uterus naklinde yer alan canlı donör ameliyatı 10 saatten fazla sürer ve uterus nakledildikten sonra uterus reddini önlemek için alıcıya yıllarca immünosüpresif ilaç tedavisi uygulanır. Immünosüpresif ilaçlar; yüksek düzeyli böbrek toksisitesi, ciddi enfeksiyon riski ve diyabet dahil olmak üzere önemli yan etkilerle ilişkilidir. Bu nedenle, uterin faktörü kısırlığını tedavi etmek için alternatif yaklaşımlar geliştirilmelidir.
\end{abstract}

Anahtar sözcükler: Kısırlık, uterin faktörü, uterus nakli.

\section{ABSTRACT}

Successful births have been performed thanks to uterus transplantations to recipients from living donors. Females who are infertile due to nonfunctional uterus can be treated. The live donor operation included in uterus transplantation lasts for more than 10 hours and the recipient is administered immunosuppressive drug treatment for years after the transplantation of the uterus to prevent the rejection of the uterus. Immunosuppressive drugs are associated with significant side effects including high-level kidney toxicity, severe infection risk, and diabetes. For this reason, alternative approaches should be developed to treat uterine factor infertility.

Keywords: Infertility, uterine factor, uterine transplantation.

\section{UTERUS NAKLI İÇIN FAKTÖRLER}

Bir bireyde doğuştan uterus (rahim) bulunmayabilir, hastalık nedeniyle uterusu cerrahi yolla alınmış olabilir ya da uterus görevini yapmayabilir. Tüm bunlar mutlak uterin faktör kısırlığının ve uterus naklinin sebebidir.

Ylllarca mutlak uterin faktör kısırlığının tedavisi olmadığı düşünülmüştür. İlerleyen bilim ve teknoloji ile beraber uterus nakli fikri ortaya çıkmıştır. ${ }^{[2]}$

\section{UTERUS NAKLI OLGULARI}

Uterus naklinin ilk klinik uygulamas1 2000 yılının Nisan ayında Suudi Arabistan'da yapılmıştır. ${ }^{[2]}$ Alıcı, doğum sonrası kanama nedeniyle altı yıl önce uterusu alınmış olan 26 yaşında bir kadın, verici (donör) ise, premenopozal dönemdeki 46 yaşında bir kadın idi. Donör histerektomisi (rahmin vücuttan tümden çıkarılması) kısa uterin damarlarla yapılmış ve damar uzantılarıyla birlikte alıcıya uç uca anastomoz edilmiştir. Ameliyattan sonra immün baskılayıcı olarak siklosporin, kortikosteroid ve azatiyoprin kullanılmıştır. Organ nakli ameliyatının kendisi başarılı olsa da, uterus nekrozuna bağlı oluşan pihtı nedeniyle nakledilen uterus, ameliyat sonrası 99. günde cerrahi yolla çıkarılmıştır. İlk uterus nakli denemesi başarısızlıkla sonuçlanmasına rağmen

İletişim adresi: Mine Ün. İstanbul Aydın Üniversitesi Tıp Fakültesi Öğrencisi, 34295 Sefaköy, Küçükçekmece, İstanbul, Türkiye.

e-posta: unmine13@gmail.com 
uterus nakli konusundaki girişimleri önemli ölçüde ilerletmiştir. ${ }^{[3]}$

$\mathrm{Bu}$ denemeden sonra uterus naklindeki temel çalışmaların yetersiz olduğu düşünülmüş ve hayvan çalışmalarına ağırlık verilmiştir. ${ }^{[3]}$

Uterus nakli ile ilgili hayvan deneyleri farelerde, ${ }^{[4-8]}$ sıçanlarda, ${ }^{[9-14]}$ tavşanlarda, ${ }^{[15-18]}$ domuzlarda, ${ }^{[19-32]}$ maymunlarda, ${ }^{[33-40]}$ babunlar gibi insan dişındaki primatlarda ${ }^{[41-43]}$ gerçekleştirilmiştir.

Uterus naklinin ikinci klinik uygulaması Ağustos 2011'de bir Türk ekibi tarafından Türkiye'de (Akdeniz Üniversitesi Hastanesi, Antalya, Türkiye) gerçekleştirilmiştir. Verici, bir trafik kazası sonucu beyin ölümü gerçekleşmiş olan 22 yaşında, alıcı ise MRKH (Mayer Rokitansky Kuster Hauser) sendromu (doğuştan uterusun olmaması veya uterusun gelişememesi) görülen 21 yaşında bir kadın idi. Vericinin ortak iliyaklarının dış iliyak damarlarına iki taraflı uçtan yana anastomozu dahil nakil altı saat sürmüş ve günlük timoglobulin 10 gün boyunca verilmiş ve bunu takrolimus ile prednizolon takip etmiştir. Dört yıldan uzun süren çoklu embriyo transferi girişimlerine rağmen, başarılı bir hamilelik görülmemiştir. Gebelikte başarısızlığın nedeni açık değildir, fakat embriyoya özgü faktörlerden ziyade uterusa özgü faktörlere

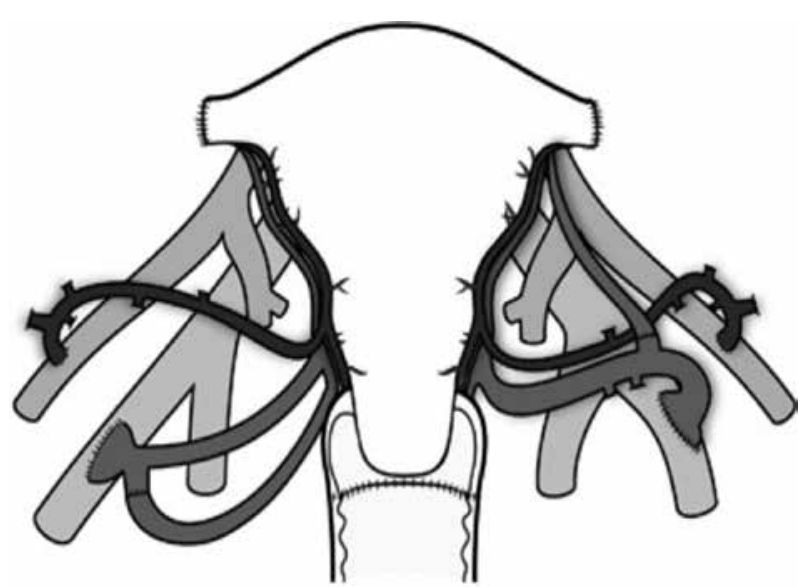

Şekil 1. Canlı donörlerden alıcıya uterus naklinde vasküler bağlantıların şematik çizimi. Vericinin internal iliyak arterlerin ön dalları ve internal iliyak venlerinin segmentleri, alıcının eksternal iliyak damarlarına yan yana anastomoz edilir. Sol tarafta, yumurtalık veniyle anatomik olarak birleşen üst uterin veni, venöz çıkığı artırmak için vericinin internal iliyak venine anastomoz edilmiştir. Sağ tarafta, yumurtalık damarlarından biri, vericinin ureterine zarar vermeden çıkarılması için organ alımından önce kesilmiştir. ${ }^{[50]}$ bağlı olduğu düşünülmektedir. Çünkü alıcı da verici de hamile kalabilecek yeteneğe sahip ve genç yaştaydı. Uterus bugün hala alıcıda bulunmaktadır. ${ }^{[44-47]}$

İsveç'te, 2013'ün başlarında dokuz canlı donörden uterus nakli gerçekleştirilmiştir. ${ }^{[48]}$ Sekiz alıcıda MRKH sendromu vardı, dokuzuncuya ise daha önce rahim ağzı kanseri nedeniyle histerektomi yapılmıştı. Alıcılardan beşinin annesi, birinin teyzesi, birinin kayınvalidesi, birinin yakın arkadaşı donör idi. ${ }^{[49]}$ Donör ameliyatı bir orta hat kesisi ile yapılmıştır. Uterus, üreterlere ve bitişik uterus damarlarına zarar gelmesi önlenerek ana uterin venleri içerecek şekilde alınmıştır. Donör ameliyat1 10.5-13 saat sürmüş ve tüm donörler altı gün hastanede kalmış, hiçbir donörde doku bozukluğu saptanmamıştır. ${ }^{[48]}$ Uterus donörden alındıktan sonra dışarıda yıkanmış, soğutulmuş, ardından çift taraflı uçtan yana dış iliyaklara yapılan anastomozlarla ve vajinal anastomozla alıcıya nakledilmiştir (Şekil 1). ${ }^{[48,50]}$ İmmün baskılayıc1 olarak iki doz timoglobulin, metilprednizolon ile verilmiş ve bakım tedavisi takrolimus ile yapılmıştır. Illk aylarda iki hastaya uterus damarlarında oluşan iki taraflı pihtı nedeniyle histerektomi yapilması gerekmiştir.

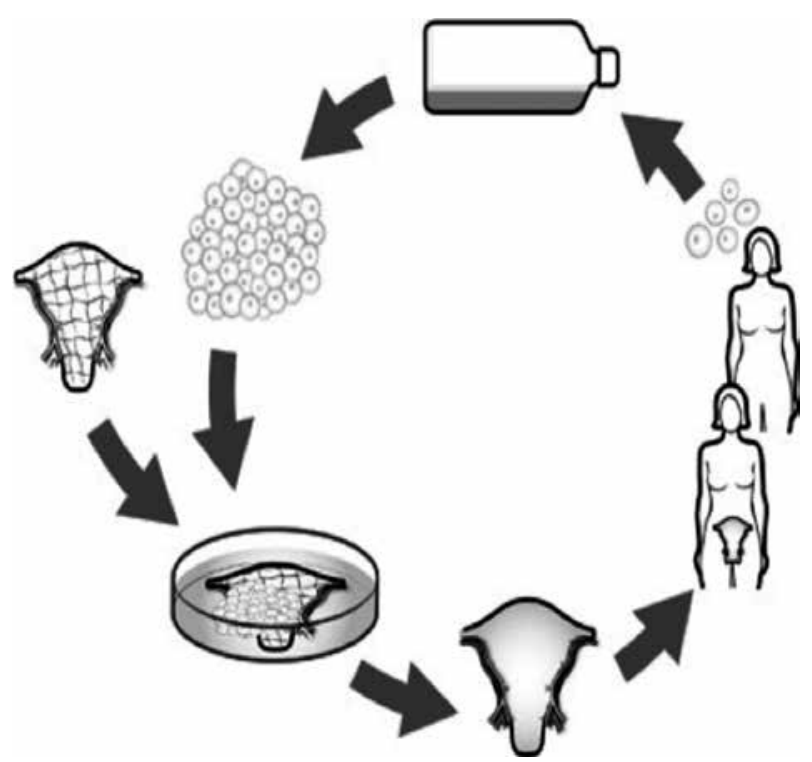

Şekil 2. Biyomühendislikte uterusun oluşturulma aşamaları. Alıcının kök hücreleri in vitro olarak çoğaltılır. Biyoreaktiflerle uygun ortamda organ haline getirilir. Biyomühendislik ürünü organ daha sonra alıcıya nakledilir, alıcı herhangi bir immünosüpresif ilaca ihtiyaç duymaz ve organı uzun yıllar yan etkisi olmadan taşıyabilir. ${ }^{[50]}$ 
Bu iki başarısızlık, nakilde 60 yaş üstü donörler kullanılan ve aynı zamanda nakilden hemen sonra en düşük kan akışı olan üç uterustan ikisinde meydana gelmiştir. Bu faktörler donör başarısızlıkları ile ilişkili olabilir. ${ }^{[48]}$ Kalan yedi alıcı, uterus naklinden 1-2 ay sonra kendiliğinden ve düzenli adet görmüştür. ${ }^{[42]}$ Embriyo transferleri uterus naklinden 12-14 ay sonra yapılmıştır. Mart 2014 'ten itibaren dondurulmuş embriyolar açılmış ve alıcılara transfer edilmiştir. İki alıcı ilk embriyo transferi denemelerinde gebe kalmış ve uterus naklinden sonraki ilk canlı doğum 4 Eylül 2014'te gerçekleşmiştir. Böylece dünyanın ilk uterus nakli bebeği İsveç'te doğmuştur. ${ }^{[48,51]}$

$\mathrm{Bu}$ çalışmaları takiben Nisan 2017 resmi raporuna göre beş doğum gerçekleşmiştir. ${ }^{[52]}$

\section{BIYYTEKNOLOJIK BIR UTERUS OLUŞTURULMASI}

Son yillarda, gelecekteki uterus nakilleri için biyolojik olarak yapilandırılmış rahim oluşturmak amaçlanmış ve bu amaçla projeler başlatılmıştır. Bu projelerin asıl hedefi organ bulma sıkıntısını aşmak, canlı bir donör ameliyatına gerek duymamayı sağlamak ve alıcının nakledilen uterusa karşı immün yanıt oluşturmasını engellemektir. En önemlisi de bahsedilen son maddedir. Çünkü biyoteknolojik olarak yapilacak olan uterus alıcının kendi kök hücresinden oluşturulacaktır. $\mathrm{Bu}$ da alıcının immün sisteminin tepki verme ihtimalini ve alıcının immünosüpresif ilaçlara maruz kalma sorununu ortadan kaldıracaktır (Şekil 2). ${ }^{[1,50]}$

Eğer yapay uterus yapilabilirse, uterin faktör kısırlığı tedavisi için uterus nakli gereksiz kalacaktır. ${ }^{[53]}$

\section{UTERUS NAKLINE İLIŞKIN ETIK, YASAL VE SOSYAL SORUNLAR}

Uterus nakli iki yönden tıbbi bir tedavidir: (i) üreme tıbbı ve (ii) organ nakli. Ancak yaşamı tehdit eden bir organ olmadığı için organ naklinde etiksel sorunlarla karşılaşılmaktadır. 1997'de yürürlüğe giren Organ Nakli Yasasina göre organ nakli ölen bağışçının kalbini, akciğerleri, karaciğerini, pankreasını, böbreklerini ve ince bağırsağını içerir. Bununla birlikte, canlı bağışçılardan yapilan nakil uygulaması sadece etik kurallarla düzenlenir. Diğer bir deyişle, bugün uterus naklinin yasadışı olmaması için bağışçının canlı olması gerekmektedir. Bu yüzden kapsamlı bir yasal gelişme gereklidir. ${ }^{[54]}$

Uterus naklinin klinik uygulamasıyla ilgili birçok etik sorun olmasına rağmen, Japonya başta olmak üzere birçok ülkenin vatandaşlarının yaklaşık \%80'i uterus naklini etik olarak kabul etmiştir. ${ }^{55-63]}$

\section{Çıkar çakışması beyanı}

Yazar bu yazının hazırlanması ve yayınlanması aşamasında herhangi bir çıkar çakışması olmadığını beyan etmiştir.

\section{Finansman}

Yazar bu yazının araştırma ve yazarlık sürecinde herhangi bir finansal destek almadıklarını beyan etmiştir.

\section{KAYNAKLAR}

1. Brännström M. Uterus transplantation and beyond. J Mater Sci Mater Med 2017;28:70.

2. Fageeh W, Raffa H, Jabbad H, Marzouki A. Transplantation of the human uterus. Int $\mathrm{J}$ Gynaecol Obstet 2002;76:245-51.

3. Kandela P. Uterine transplantation failure causes Saudi Arabian government clampdown. Lancet 2000;356:838.

4. Racho El-Akouri R, Kurlberg G, Dindelegan G, Mölne $\mathrm{J}$, Wallin A, Brännström M. Heterotopic uterine transplantation by vascular anastomosis in the mouse. J Endocrinol 2002;174:157-66.

5. Racho El-Akouri R, Kurlberg G, Brännström M. Successful uterine transplantation in the mouse: pregnancy and post-natal development of offspring. Hum Reprod 2003;18:2018-23.

6. Racho El-Akouri R, Wranning CA, Mölne J, Kurlberg G, Brännström M. Pregnancy in transplanted mouse uterus after long-term cold ischaemic preservation. Hum Reprod 2003;18:2024-30.

7. El-Akouri RR, Mölne J, Groth K, Kurlberg G, Brännström M. Rejection patterns in allogeneic uterus transplantation in the mouse. Hum Reprod 2006;21:436-42.

8. Wranning CA, El-Akouri RR, Groth K, Mölne $\mathrm{J}$, Parra AK, Brännström M. Rejection of the transplanted uterus is suppressed by cyclosporine A in a semi-allogeneic mouse model. Hum Reprod 2007;22:372-9.

9. Jiga LP, Lupu CM, Zoica BS, Ionac M. Experimental model of heterotopic uterus transplantation in the laboratory rat. Microsurgery 2003;23:246-50.

10. Wranning CA, Akhi SN, Kurlberg G, Brännström $M$. Uterus transplantation in the rat: model development, surgical learning and morphological 
evaluation of healing. Acta Obstet Gynecol Scand 2008;87:1239-47.

11. Wranning CA, Akhi SN, Diaz-Garcia C, Brännström M. Pregnancy after syngeneic uterus transplantation and spontaneous mating in the rat. Hum Reprod 2011;26:553-8.

12. Akhi SN, Diaz-Garcia C, El-Akouri RR, Wranning $\mathrm{CA}$, Mölne $\mathrm{J}$, Brännström $\mathrm{M}$. Uterine rejection after allogeneic uterus transplantation in the rat is effectively suppressed by tacrolimus. Fertil Steril 2013;99:862-70.

13. Díaz-García C, Akhi SN, Martínez-Varea A, Brännström $\mathrm{M}$. The effect of warm ischemia at uterus transplantation in a rat model. Acta Obstet Gynecol Scand 2013;92:152-9.

14. Díaz-García C, Johannesson L, Shao R, Bilig H, Brännström M. Pregnancy after allogeneic uterus transplantation in the rat: perinatal outcome and growth trajectory. Fertil Steril 2014;102:1545-52.

15. SieunarineK, DoumplisD, KuzminE, Corless DJ, Hakim NS, Del Priore G, et al. Uterine allotranspiantation in the rabbit model using a macrovascular patch technique. Int Surg 2008;93:288-94.

16. Saso S, Hurst S, Chatterjee J, Kuzmin E, Thum Y, David AL, et al. Test of long-term uterine survival after allogeneic transplantation in rabbits. J Obstet Gynaecol Res 2014;40:754-62.

17. Saso S, Petts G, Chatterjee J, Thum MY, David AL, Corless $\mathrm{D}$, et al. Uterine allotransplantation in a rabbit model using aorto-caval anastomosis: a long-term viability study. Eur J Obstet Gynecol Reprod Biol 2014;182:185-93.

18. Saso S, Petts G, David AL, Thum MY, Chatterjee $\mathrm{J}$, Vicente JS, et al. Achieving an early pregnancy following allogeneic uterine transplantation in a rabbit model. Eur J Obstet Gynecol Reprod Biol 2015;185:164-9.

19. Sieunarine K, Zakaria FB, Boyle DC, Corless DJ, Noakes DE, Lindsay I, et al. Possibilities for fertility restoration: a new surgical technique. Int Surg 2005;90:249-56.

20. Wranning CA, El-Akouri RR, Lundmark C, DahmKähler P, Mölne J, Enskog A, et al. Auto-transplantation of the uterus in the domestic pig (Sus scrofa): Surgical technique and early reperfusion events. J Obstet Gynaecol Res 2006;32:358-67.

21. Sieunarine K, Boyle DC, Corless DJ, Noakes DE, Ungar L, Marr CE, et al. Pelvic vascular prospects for uterine transplantation. Int Surg 2006;91:217-22.

22. Avison DL, DeFaria W, Tryphonopoulos P, Tekin A, Attia GR, Takahashi $\mathrm{H}$, et al. Heterotopic uterus transplantation in a swine model. Transplantation 2009;88:465-9.

23. Hurst SA, Smith JR, Del Priore G. Experiences in uterine transplantation. Transplantation 2010;89:769.

24. Ramirez ER, Ramirez DK, Pillari VT, Vasquez H, Ramirez HA. Modified uterine transplant procedure in the sheep model. J Minim Invasive Gynecol 2008;15:311-4.

25. Wranning CA, Dahm-Kähler P, Mölne J, Nilsson UA, Enskog A, Brännström M. Transplantation of the uterus in the sheep: oxidative stress and reperfusion injury after short-time cold storage. Fertil Steril 2008;90:817-26.

26. Dahm-Kähler P, Wranning C, Lundmark C, Enskog A, Mölne J, Marcickiewicz J, et al. Transplantation of the uterus in sheep: methodology and early reperfusion events. J Obstet Gynaecol Res 2008;34:784-93.

27. Dittrich R, Maltaris T, Mueller A, Strahl O, Hoffmann I, Beckmann MW, et al. Uterus cryopreservation in the sheep: one step closer to uterus transplantation. In Vivo 2010;24:629-34.

28. Ramirez ER, Ramirez Nessetti DK, Nessetti MB, Khatamee M, Wolfson MR, Shaffer TH, et al. Pregnancy and outcome of uterine allotransplantation and assisted reproduction in sheep. $\mathrm{J}$ Minim Invasive Gynecol 2011;18:238-45.

29. Gauthier T, Bertin F, Fourcade L, Maubon A, Saint Marcoux F, Piver P, et al. Uterine allotransplantation in ewes using an aortocava patch. Hum Reprod 2011;26:3028-36.

30. Gonzalez-Pinto IM, Tryphonopoulos P, Avison DL, Nishida S, Tekin A, Santiago S, et al. Uterus transplantation model in sheep with heterotopic whole graft and aorta and cava anastomoses. Transplant Proc 2013;45:1802-4.

31. Wei L, Xue T, Yang H, Zhao GY, Zhang G, Lu $\mathrm{ZH}$, et al. Modified uterine allotransplantation and immunosuppression procedure in the sheep model. PLoS One 2013;8:81300.

32. Saso S, Petts G, Thum MY, Corless D, Boyd M, Noakes D, et al. Achieving uterine auto-transplantation in a sheep model using iliac vessel anastomosis: a short-term viability study. Acta Obstet Gynecol Scand 2015;94:245-52.

33. Mihara M, Kisu I, Hara H, Iida T, Yamamoto $\mathrm{T}$, Araki J, et al. Uterus autotransplantation in cynomolgus macaques: intraoperative evaluation of uterine blood flow using indocyanine green. Hum Reprod 2011;26:3019-27.

34. Kisu I, Banno K, Mihara M, Lin LY, Tsuji K, Yanokura $\mathrm{M}$, et al. Indocyanine green fluorescence imaging for evaluation of uterine blood flow in cynomolgus macaque. PLoS One 2012;7:35124.

35. Mihara M, Kisu I, Hara H, Iida T, Araki J, Shim T, et al. Uterine autotransplantation in cynomolgus macaques: the first case of pregnancy and delivery. Hum Reprod 2012;27:2332-40.

36. Kisu I, Mihara M, Banno K, Hara H, Masugi Y, Araki J, et al. Uterus allotransplantation in cynomolgus macaque: a preliminary experience with non-human primate models. J Obstet Gynaecol Res 2014;40:907-18.

37. Kisu I, Banno K, Mihara M, Hara H, Umene K, Adachi $\mathrm{M}$, et al. A surgical technique using the ovarian vein in non-human primate models of potential living-donor surgery of uterus transplantation. Acta Obstet Gynecol Scand 2015;94:942-8. 
38. Obara H, Kisu I, Kato Y, Yamada Y, Matsubara K, Emoto $\mathrm{K}$, et al. Surgical technique for allogeneic uterus transplantation in macaques. Sci Rep 2016;6:35989.

39. Adachi M, Kisu I, Nagai T, Emoto K, Banno K, Umene $\mathrm{K}$, et al. Evaluation of allowable time and histopathological changes in warm ischemia of the uterus in cynomolgus monkey as a model for uterus transplantation. Acta Obstet Gynecol Scand 2016;95:991-8.

40. Kisu I, Kato Y, Yamada Y, Matsubara K, Obara $\mathrm{H}$, Emoto $\mathrm{K}$, et al. Organ Perfusion for Uterus Transplantation in Non-Human Primates With Assumed Procurement of a Uterus From a Brain-Dead Donor. Transplant Proc 2016;48:1266-9.

41. Enskog A, Johannesson L, Chai DC, Dahm-Kähler P, Marcickiewicz J, Nyachieo A, et al. Uterus transplantation in the baboon: methodology and longterm function after auto-transplantation. Hum Reprod 2010;25:1980-7.

42. Johannesson L, Enskog A, Dahm-Kähler P, Hanafy A, Chai DC, Mwenda JM, et al. Uterus transplantation in a non-human primate: long-term follow-up after autologous transplantation. Hum Reprod 2012;27:1640-8.

43. Johannesson L, Enskog A, Mölne J, Diaz-Garcia C, Hanafy A, Dahm-Kähler $P$, et al. Preclinical report on allogeneic uterus transplantation in non-human primates. Hum Reprod 2013;28:189-98.

44. Ozkan O, Akar ME, Ozkan O, Erdogan O, Hadimioglu $\mathrm{N}$, Yilmaz $\mathrm{M}$, et al. Preliminary results of the first human uterus transplantation from a multiorgan donor. Fertil Steril 2013;99:470-6.

45. Ozkan O, Akar ME, Erdogan O, Ozkan O, Hadimioglu N. Uterus transplantation from a deceased donor. Fertil Steril 2013;100:41.

46. Erman Akar M, Ozkan O, Aydinuraz B, Dirican $\mathrm{K}$, Cincik M, Mendilcioglu I, et al. Clinical pregnancy after uterus transplantation. Fertil Steril 2013;100:1358-63.

47. Akar ME, Ozkan O, Ozekinci M, Sindel M, Yildirim F, Oguz N. Uterus retrieval in cadaver: technical aspects. Clin Exp Obstet Gynecol 2014;41:293-5.

48. Brännström M, Johannesson L, Dahm-Kähler P, Enskog A, Mölne J, Kvarnström N, et al. First clinical uterus transplantation trial: a six-month report. Fertil Steril 2014;101:1228-36.

49. Järvholm S, Johannesson L, Brännström M. Psychological aspects in pre-transplantation assessments of patients prior to entering the first uterus transplantation trial. Acta Obstet Gynecol Scand 2015;94:1035-8.

50. Brännström M. Uterus transplantation and beyond. J Mater Sci Mater Med 2017;28:70.

51. Brännström $M$, Johannesson L, Bokström $H$,
Kvarnström N, Mölne J, Dahm-Kähler P, et al. Livebirth after uterus transplantation. Lancet 2015;385:607-16.

52. Brännström M. The Swedish uterus transplantation project: the story behind the Swedish uterus transplantation project. Acta Obstet Gynecol Scand 2015;94:675-9.

53. Suganuma N, Hayashi A, Kisu I, Banno K, Hara $\mathrm{H}$, Mihara M. Uterus transplantation: Toward clinical application in Japan. Reprod Med Biol 2017;16:305-13.

54. Serour GI, Dickens BM. Assisted reproduction developments in the Islamic world. Int $\mathrm{J}$ Gynaecol Obstet 2001;74:187-93.

55. Semba Y, Chang C, Hong H, Kamisato A, Kokado M, Muto K. Surrogacy: donor conception regulation in Japan. Bioethics 2010;24:348-57.

56. Grynberg M, Ayoubi JM, Bulletti C, Frydman R, Fanchin R. Uterine transplantation: a promising surrogate to surrogacy? Ann N Y Acad Sci 2011;1221:47-53.

57. Robertson JA. Other women's wombs: uterus transplants and gestational surrogacy. J Law Biosci 2016;3:68-86.

58. Testa G, Koon EC, Johannesson L. Living Donor Uterus Transplant and Surrogacy: Ethical Analysis According to the Principle of Equipoise. Am J Transplant 2017;17:912-6.

59. Hayashi A, Hirai S, Tsutsumishita Y, Kisu I, Mihara M, Suganuma N. Awareness Survey on Clinical Application of Uterus Transplantation among General Public [Abstract]. Paper presented at the American Society for Reproductive Medicine 2014 Annual Meeting; October 18-22, 2014; Honolulu, HI; 2014.

60. Hayashi A, Tsutsumishita Y, Kisu I, Mihara M, Suganuma N. Sequential Awareness Surveys on Clinical Application of Uterine Transplantation Among Japanese General Public [Abstract]. Paper presented at the 31st Annual Meeting of the European Society of Human Reproduction and Embryology; June 14-17, 2015; Lisbon; 2015.

61. Kisu I, Banno K, Soeda E, Kurihara Y, Okushima M, Yamaguchi A, et al. Survey of Attitudes toward Uterus Transplantation among Japanese Women of Reproductive Age: A Cross-Sectional Study. PLoS One 2016;11:e0156179.

62. Wennberg AL, Rodriguez-Wallberg KA, Milsom I, Brännström M. Attitudes towards new assisted reproductive technologies in Sweden: a survey in women 30-39 years of age. Acta Obstet Gynecol Scand 2016;95:38-44.

63. Saso S, Clarke A, Bracewell-Milnes T, Al-Memar M, Hamed AH, Thum MY, et al. Survey of perceptions of health care professionals in the United Kingdom toward uterine transplant. Prog Transplant 2015;25:56-63. 